International Journal of Modern Physics A

(C) World Scientific Publishing Company

\title{
SCALE OF MASS GENERATION FOR MAJORANA NEUTRINOS *
}

\author{
Hong-Jian He and Duane A. Dicus \\ Department of Physics, University of Texas at Austin, TX 78712, USA
}

Received: 1 November, 2004

\begin{abstract}
Scales of mass generation for Majorana neutrinos (as well as quarks and leptons) can be probed from high energy $2 \rightarrow n$ inelastic scattering involving a multiple longitudinal gauge boson final state. We demonstrate that the unitarity of $2 \rightarrow n$ scattering puts the strongest new upper limit on the scale of fermion mass generation, independent of the electroweak symmetry breaking scale $v=\left(\sqrt{2} G_{F}\right)^{-\frac{1}{2}}$. Strikingly, for Majorana neutrinos (quarks and leptons), we find that the strongest $2 \rightarrow n$ limits fall in a narrow range, $136-170 \mathrm{TeV}(3-107 \mathrm{TeV})$ with $n=20-24(n=2-12)$, depending on the observed fermion masses. Physical implications are discussed.

Keywords: Fermion Mass Generation; Majorana Neutrinos; Unitarity.
\end{abstract}

\section{Scale of Fermion Mass Generation: the Puzzle}

The standard model (SM) hypothesizes a fundamental Higgs boson to generate masses for all elementary particles: weak gauge bosons, quarks and leptons. The masses $M_{W, Z}$ of gauge bosons $\left(W^{ \pm}, Z^{0}\right)$ involve the electroweak gauge couplings $\left(g, g^{\prime}\right)$ and the vacuum expectation value $v=\left(\sqrt{2} G_{F}\right)^{-\frac{1}{2}}$, while the quark/lepton masses arise from the product of Yukawa couplings $y_{f}$ and $v$. Unlike gauge interaction, the flavor-dependent Yukawa couplings $y_{f}$ are completely arbitrary, exhibiting a large hierarchy $y_{e} / y_{t}=m_{e} / m_{t} \simeq 3 \times 10^{-6}$ between the electron and top quark. No compelling principle requires the fermion mass generation to share the same mechanism as the $W / Z$ gauge bosons. Moreover, the tiny neutrino masses $m_{\nu} \gtrsim 0.05 \mathrm{eV}$ could not be generated by such a Higgs boson without losing renormalizability $1 \frac{1}{\text { or }}$ extending the SM particle spectrum 23. So far, neither is the Higgs boson found nor is any Yukawa coupling experimentally measured - the origin and scale of mass generation remain, perhaps, the greatest mystery.

What is wrong with just putting all the bare masses into the SM Lagrangian by hand? These bare mass-terms can be made gauge invariant in the nonlinear realization 4 , but are manifestly nonrenormalizable. This leads to unitarity violation in high energy scattering at a scale $E^{\star}$. Generically, we define the scale $\Lambda_{x}$ for

*Talk presented by H.J.H. at the Conference of the Division of Particles and Fields, American Physical Society (DPF-2004), held at University of California, Riverside, CA, USA, August 26-31, 2004. 
generating a mass $m_{x}$ to be the minimal energy above which the bare mass term for $m_{x}$ has to be replaced by a renormalizable interaction (adding at least one new physical state to the particle spectrum). Hence the unitarity violation scale $E^{\star}$ puts a universal upper bound on $\Lambda_{x}$, i.e., $\Lambda_{x} \leqslant E^{\star}$.

A mass term for the bare masses $M_{W, Z}$ will cause the high energy $2 \rightarrow 2$ longitudinal gauge boson scattering to violate unitarity at a scale, $\underline{56718}$

$$
E_{W}^{\star} \simeq \sqrt{8 \pi} v \simeq 1.2 \mathrm{TeV} .
$$

This puts an upper limit on the scale of the electroweak symmetry breaking (EWSB) and justifies TeV energy scale for the construction of the CERN LHC. Similarly, with the bare mass terms for Dirac fermions (quarks and leptons), an upper bound on the scale of fermion mass generation can be derived from the $2 \rightarrow 2$ inelastic scattering $f_{ \pm} \bar{f}_{ \pm} \rightarrow V_{L}^{a_{1}} V_{L}^{a_{2}}\left(V^{a}=W^{ \pm}, Z^{0}\right), \underline{9}$

$$
E_{f}^{\star} \simeq \frac{8 \pi v^{2}}{\sqrt{N_{c}} m_{f}}
$$

where $N_{c}=3(1)$ for quarks (leptons). This shows that the upper limit on the scale of fermion mass generation is proportional to $1 / m_{f}$ and is thus independent of the classic bound $E_{W}^{\star}$ on the EWSB scale. Also, for all the SM fermions (except the top quark), the limit (2) is substantially higher than $E_{W}^{\star}$. For the scale of mass generation for Majorana neutrinos, Refs. 10 11] derived an analogous $2 \rightarrow 2$ bound,

$$
E_{\nu}^{\star} \simeq \frac{4 \pi v^{2}}{m_{\nu}}
$$

which is about $10^{16} \mathrm{GeV}$ (the GUT scale) for typical input $m_{\nu} \sim 0.05 \mathrm{eV}$ [13].

However, by considering the $2 \rightarrow n$ inelastic scattering, $f \bar{f}, \nu \nu \rightarrow n V_{L}^{a}(n>2)$, it was noted 11 that the $n$-body phase space integration contributes a large energy power factor $s^{n-2}$ to further enhance the cross section (in addition to the energy dependence $s^{1}$ from the squared amplitude), so the unitarity limit for a fermion $f(\nu)$ would behave like

$$
E_{f(\nu)}^{\star} \sim v\left(\frac{v}{m_{f(\nu)}}\right)^{\frac{1}{n-1}} \longrightarrow v, \quad(\text { for } n \rightarrow \text { large }),
$$

which could be pushed arbitrarily close to the weak scale $v$ and thus become independent of the fermion mass $m_{f}$ for large enough $n$. This raises a deep puzzle: is there an independent new scale for fermion mass generation revealed from the fermion-(anti)fermion scattering into weak bosons? We find the behavior (4) very counter-intuitive since the kinematic condition forces any $2 \rightarrow n$ unitarity limit $E^{\star}$ to grow at least linearly with $n$ [12,

$$
\sqrt{s}>n M_{W(Z)} \simeq \frac{n}{3} v, \quad \longrightarrow \frac{E^{\star}}{v}>\frac{n}{3} .
$$




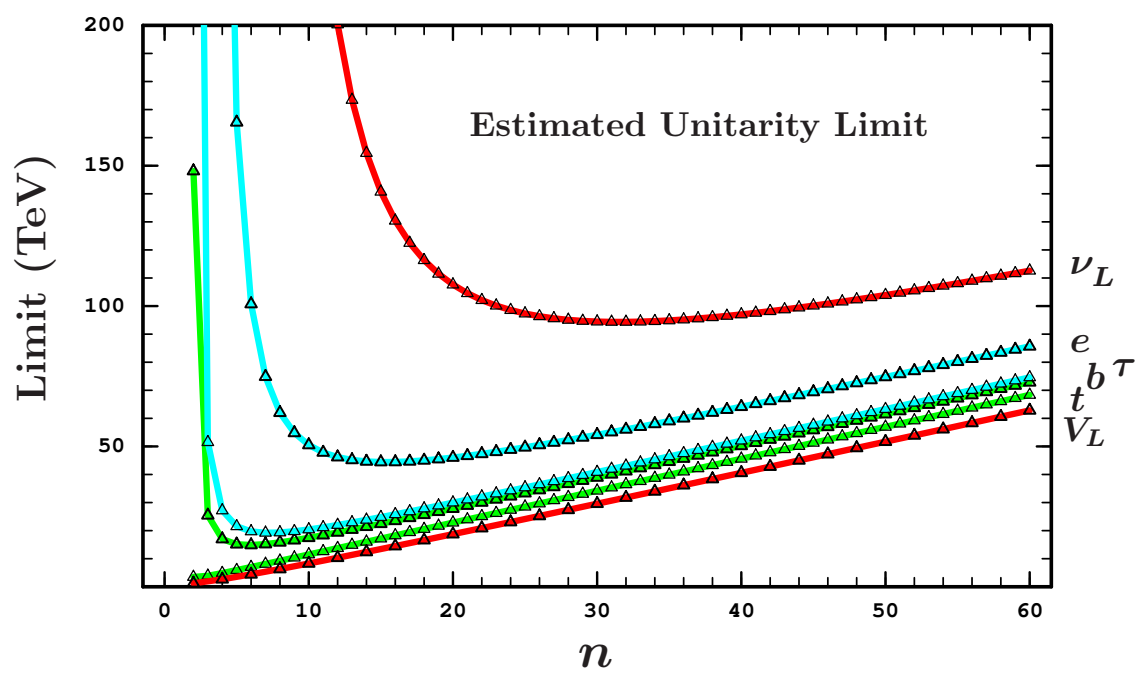

Fig. 1. Realistic estimate of the unitarity bound $E^{\star}$ for the scattering processes $V_{L}^{a_{1}} V_{L}^{a_{2}}, t \bar{t}, b \bar{b}$, $\tau^{-} \tau^{+}, e^{-} e^{+}, \nu_{L} \nu_{L} \rightarrow n V_{L}^{a}$ (curves from bottom to top), as a function of $n(\geqslant 2)$.

\section{The Resolution and New Upper Limits}

\subsection{The Resolution}

We observe that the resolution to this puzzle has to rely on the additional $n$ dependent dimensionless factors in the exact n-body phase space integration which can sufficiently suppress the $E$-power enhancement $s^{n-2}$ mentioned above Eq. (4). Computing the exact $n$-body phase space we can formally derive the $2 \rightarrow n$ unitarity limit as follows, 12

$$
E^{\star}=v\left[C_{0} 2^{4 n-2} \pi^{2(n-1)} \varrho(n-1) !(n-2) !\left(2 N_{c}\right)^{\delta-2}\left(\frac{v}{m_{f, \nu}}\right)^{2(2-\delta)}\right]^{\frac{1}{2(n-2+\delta)}}
$$

where $\delta=1(2)$ for the scattering $f \bar{f}, \nu_{L} \nu_{L}\left(V_{L}^{a_{1}} V_{L}^{a_{2}}\right) \rightarrow n V_{L}^{a}$, $\varrho$ is the symmetry factor from the identical particles in the final state, and the constant $C_{0}$ originates from the dimensionless coefficient in the squared amplitude (whose possible angular dependence is included in the exact $n$-body phase space integration). Since all large $n$ factors are explicitly counted in [6], we expect that, independent of the detail, $C_{0}^{\frac{1}{2(n-2+\delta)}} \sim 1$ reasonably holds and becomes increasingly more accurate as $n$ gets larger. So, setting $C_{0} \simeq 1$ and $\varrho \simeq 1$ for simplicity we deduce an realistic estimate of the unitarity limit $E^{\star}$ shown in Fig. 1 (where $m_{\nu}=0.05 \mathrm{eV}$ is chosen). Using the Stirling formula $n ! \simeq n^{n} e^{-n} \sqrt{2 \pi n}$, we can then derive the correct asymptotic behavior from (6),

$$
E^{\star} \rightarrow v \frac{4 \pi n}{e}>v \frac{n}{3}, \quad(\text { for } n \gg 1),
$$




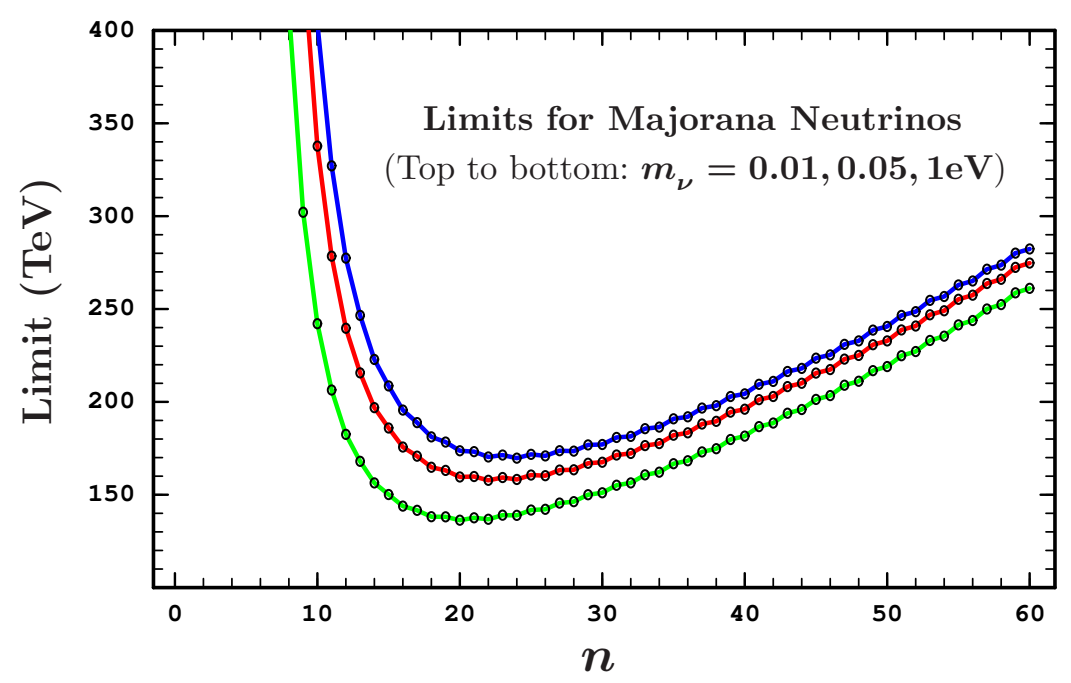

Fig. 2. Precise unitarity limit $E_{\nu}^{\star}$ of Majorana neutrinos from the scattering processes $\nu_{L} \nu_{L} \rightarrow$ $n V_{L}^{a}$ (curves from bottom to top), as a function of $n(\geqslant 2)$.

in full agreement with the kinematic condition (5).

We see that for $V_{L}^{a_{1}} V_{L}^{a_{2}}$ and $t \bar{t}$ initial states the strongest bound (minimum of the curve) still occurs at $n=n_{s}=2$; while for all light fermions including Majorana neutrinos the best limit for the scale of mass generation lies at a new minimum with $n=n_{s}>2$ and $E^{\star}$ no higher than about $100 \mathrm{TeV}$, which is substantially tighter than the corresponding classic $2 \rightarrow 2$ bound. As we observed $\frac{12}{2}$, it is the competition between the large asymptotic linear growth (7) and the strong power suppression (44) that generates a genuine new minimum scale $E_{\min }^{\star}$ for all light fermions at $n=n_{s}>2$, independent of the EWSB scale.

\subsection{Scale of Mass Generation for Majorana Neutrinos}

For Majorana neutrinos, the dimension-3 bare mass term can be written as $-\frac{1}{2} m_{\nu}^{i j} \nu_{L i}^{T} \widehat{C} \nu_{L j}+$ H.c., which takes the gauge-invariant nonlinear form

$$
\mathcal{L}_{\nu}=-\frac{1}{2} m_{\nu}^{i j} L_{i}^{\alpha T} \widehat{C} L_{j}^{\beta} \bar{\Phi}^{\alpha^{\prime}} \bar{\Phi}^{\beta^{\prime}} \epsilon^{\alpha \alpha^{\prime}} \epsilon^{\beta \beta^{\prime}}+\text { H.c. }
$$

where $\widehat{C}=i \gamma^{2} \gamma^{0}, \bar{\Phi}=U(0,1)^{T}, U=\exp \left[i \pi^{a} \tau^{a} / v\right]$ and $L_{j}=\left(\nu_{L j} / \sqrt{2}, \ell_{L j}\right)^{T}$. We then quantitatively compute the high energy scattering $\nu_{L} \nu_{L} \rightarrow n V_{L}^{a}\left(n \pi^{a}\right)$ by using the equivalence theorem 6814] The leading amplitude $\nu_{L} \nu_{L} \rightarrow n \pi^{a}$ is given by the $\nu_{L}-\nu_{L}-\pi^{n}$ contact interactions in (8) and is of $O\left(m_{\nu} E / v^{n}\right)$ by power counting 15. Defining a real Majorana neutrino field $\chi=\left(\nu_{L}+\nu_{L}^{c}\right) / \sqrt{2}$, we compute the scattering amplitude of $\frac{1}{\sqrt{2}}\left[\left|\chi_{j+} \bar{\chi}_{j+}\right\rangle \mp\left|\chi_{j-} \bar{\chi}_{j-}\right\rangle\right] \rightarrow\left(\pi^{+}\right)^{\ell}\left(\pi^{-}\right)^{\ell}\left(\pi^{0}\right)^{n-2 \ell}$ for three cases: (a). $n$ (even) $=2 l$; (b). $n$ (even) $>2 l$; (c). $n$ (odd) $\geqslant 3$. Thus, we derive 
the unitarity limit, 12

$$
E_{\nu}^{\star}=v\left[\left(\frac{v}{m_{\nu j}}\right)^{2} \frac{4 \pi}{\mathcal{R}_{\nu k}^{\max }}\right]^{\frac{1}{2(n-1)}},
$$

where $\mathcal{R}_{\nu k}^{\max }$ is given by

$$
\begin{array}{ll}
\mathcal{R}_{\nu 1}^{\max }=\frac{\left(\frac{n}{2} !\right)^{2}\left(K_{1, n}\right)^{2}}{2^{3 n-4} \pi^{2 n-3}(n-1) !(n-2) !}, & \left(n=\text { even, } \ell=\frac{n}{2}\right), \\
\mathcal{R}_{\nu 2}^{\max }=\frac{n\left(K_{1, n}+K_{3, n}\right)^{2}}{2^{4(n-1)} \pi^{2 n-3}(n-2) !}, & \left(n=\text { even, } \ell<\frac{n}{2}\right), \\
\mathcal{R}_{\nu 3}^{\max }=\frac{n\left(K_{2, n}\right)^{2}}{2^{4(n-1)} \pi^{2 n-3}(n-2) !}, & (n=\text { odd }),
\end{array}
$$

and $K_{j, n}$ are functions of $n$ 12. For $n=$ even, the best limits are from $\left(\mathcal{R}_{\nu 1}^{\max }, \mathcal{R}_{\nu 2}^{\max }\right)_{\max }$, but numerically the bounds from $\mathcal{R}_{\nu 1}^{\max }$ and $\mathcal{R}_{\nu 2}^{\max }$ only differ by less than $2 \%$. As shown in Fig. 2, we find the optimal limits,

$$
E_{\min }^{\star}=136,158,170 \mathrm{TeV}, \quad \text { located at } n=n_{s}=20,22,24,
$$

for $m_{\nu j}=1,0.05,0.01 \mathrm{eV}$, respectively. This agrees with our estimate in Fig. 1 to less than about a factor of 1.7 .

\subsection{Scale of Mass Generation for Quarks and Leptons}

Similarly, we derive the quantitative $2 \rightarrow n$ bounds for Dirac fermions - the quarks and leptons. Consider a pair of SM fermions $\left(f, f^{\prime}\right)$ which forms a lefthanded $S U(2)_{L}$ doublet $F_{L}=\left(f_{L}, f_{L}^{\prime}\right)^{T}$ and two right-handed weak singlets $f_{R}$ and $f_{R}^{\prime}$. We then formulate their bare Dirac mass-terms $-m_{f} \bar{f} f-m_{f^{\prime}} \overline{f^{\prime}} f^{\prime}$ into the gauge-invariant nonlinear form,

$$
\mathcal{L}_{f}=-m_{f} \overline{F_{L}} U\left(\begin{array}{l}
1 \\
0
\end{array}\right) f_{R}-m_{f}, \overline{F_{L}} U\left(\begin{array}{l}
0 \\
1
\end{array}\right) f_{R}^{\prime}+\text { H.c. }
$$

Thus we compute the scattering amplitude for $\mid$ in $\rangle \rightarrow\left(\pi^{+}\right)^{k}\left(\pi^{-}\right)^{\ell}\left(\pi^{0}\right)^{n-k-\ell}$, where the initial state $\mid$ in $\rangle$ consists of two fermions in the color-singlet channe 12 . From a systematical analysis we derive the unitarity limit for quarks and leptons,

$$
E_{f}^{\star}=v\left[\left(\frac{v}{m_{\widehat{f}}}\right)^{2} \frac{4 \pi}{N_{c} \widehat{\mathcal{R}}_{j}^{\max }}\right]^{\frac{1}{2(n-1)}},
$$

where $\widehat{\mathcal{R}}_{j}^{\max }=\left(2 \mathcal{R}_{1}^{\max }, 2 \mathcal{R}_{2}^{\max }, \mathcal{R}_{3}^{\max }\right)$ is a function of $n$ [12]. We depict the numerical bounds $E_{f}^{\star}$ in Fig. 3, which shows that the best limits for all light fermions occur at a new minimum $n=n_{s}>2$ with $E_{b}^{\star}=24.5 \mathrm{TeV}$ for $b$ quark and $E_{e}^{\star}=107 \mathrm{TeV}$ for electron. These two limits only differ by about a factor 4 . 


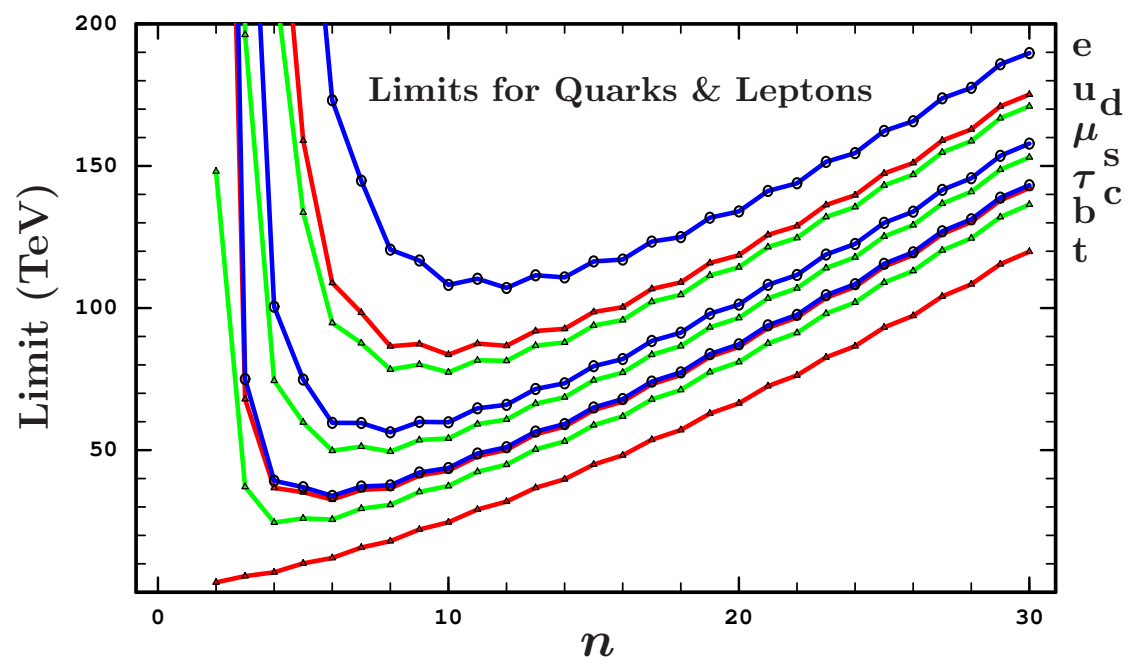

Fig. 3. Precise unitarity limits $E_{f}^{\star}$ of quarks and leptons for the scattering $t \bar{t}, b \bar{b}, c \bar{c}, \tau^{-} \tau^{+}$, $s \bar{s}, \mu^{-} \mu^{+}, d \bar{d}, u \bar{u}, e^{-} e^{+} \rightarrow n V_{L}^{a}$ (curves from bottom to top), as a function of $n(\geqslant 2)$.

\section{Discussions and Conclusions}

We have systematically analyzed the unitarity limits from the $2 \rightarrow n$ inelastic scattering $(n \geqslant 2)$, which provide a universal upper bound on the scale of mass generations for Majorana neutrinos as well as leptons and quarks. The numerical result $\$ 12$ are summarized by Table 1 , in comparison with the classic $2 \rightarrow 2$ limits 911 .

Table 1. Summary of our best unitarity limit $E_{2 \rightarrow n}^{\star}$ (in TeV) from each scattering $\xi_{1} \xi_{2} \rightarrow n V_{L}^{a}\left(n \pi^{a}\right)$, in comparison with the classic $2 \rightarrow 2$ limit $E_{2 \rightarrow 2}^{\star}(\mathrm{in} \mathrm{TeV})$.

\begin{tabular}{c||c|cccccc|ccc|c}
\hline \hline$\xi_{1} \xi_{2}$ & $V_{L}^{a_{1}} V_{L}^{a_{2}}$ & $t \bar{t}$ & $b \bar{b}$ & $c \bar{c}$ & $s \bar{s}$ & $d \bar{d}$ & $u \bar{u}$ & $\tau^{-} \tau^{+}$ & $\mu^{-} \mu^{+}$ & $e^{-} e^{+}$ & $\nu \nu$ \\
\hline \hline$n_{s}$ & 2 & 2 & 4 & 6 & 8 & 10 & 10 & 6 & 8 & 12 & 22 \\
\hline$E_{2 \rightarrow n}^{\star}$ & 1.2 & 3.5 & 25 & 33 & 49 & 77 & 84 & 34 & 56 & 107 & 158 \\
\hline \hline$E_{2 \rightarrow 2}^{\star}$ & 1.2 & 3.5 & 148 & 497 & $4 \times 10^{3}$ & $10^{5}$ & $2 \times 10^{5}$ & 605 & $10^{4}$ & $2 \times 10^{6}$ & $10^{13}$ \\
\hline \hline
\end{tabular}

Table 1 shows that the scattering $f \bar{f} \rightarrow n V_{L}^{a}(n \geqslant 2)$ does reveal an independent scale for the fermion mass generation. Our new unitarity bounds from the $2 \rightarrow n$ scattering with $n>2$ establish a new scale of mass generation for all light fermions including Majorana neutrinos and are substantially stronger than the classic $2 \rightarrow 2$ limits. In particular, for the Majorana neutrinos with typical mass values $m_{\nu}=1-0.01 \mathrm{eV}$ (as suggested by the current experiments on neutrino oscillations, neutrinoless double-beta decay and astrophysics constraints), the best upper limits on the scale of mass generation are in the range $136-170 \mathrm{TeV}$ 
(with $n=n_{s}=20-24$ ). This is only about a factor 7 weaker than the lowest bound for the light Dirac fermions (the $b$ quark) despite their huge mass hierarchy $m_{\nu} / m_{b} \approx 2 \times\left(10^{-10}-10^{-12}\right)$. Hence, these limits are very insensitive to the variation of fermion masses. Such a strong non-decoupling feature for the scale of new physics associated with light fermion mass generation is essentially due to the chiral structure of the bare fermion mass-terms, i.e., the fact that all the left-handed SM fermions are weak-doublets but their right-handed chiral partners are weak singlets (or possibly absent in the case of Majorana neutrinos with radiative mass generation), so the decoupling theorem 16 no longer applies. Finally, we have also estimated the $2 \rightarrow n$ unitarity limit on the electroweak symmetry breaking (EWSB) scale via the scattering $V_{L}^{a_{1}} V_{L}^{a_{2}} \rightarrow n V_{L}^{a}\left(\pi^{a_{1}} \pi^{a_{2}} \rightarrow n \pi^{a}\right)$ with $n \geqslant 2$, and find that the best limit remains to be $E_{W}^{\star} \simeq 1.2 \mathrm{TeV}$ with $n=n_{s}=2$, in agreement with the customary bound.

\section{Acknowledgments}

We thank S. Willenbrock for valuable discussions. This work was supported by U.S. Department of Energy under grant No. DE-FG03-93ER40757.

\section{References}

1. S. Weinberg, Phys. Rev. Lett. 43, 1566 (1979).

2. P. Minkowski, Phys. Lett. B67, 421 (1977); T. Yanagida, in Proceedings of Workshop on Unified Theories and Baryon Number in Universe, eds. O. Sawada and A. Sugamoto (KEK, Tsukuba, 1979), p.95; M. Gell-Mann, P. Ramond, R. Slansky, in Proceedings of Workshop on Supergravity, eds. F. van Nieuwenhuizen and D. Freedman (Amsterdam, 1979), p.315; S. L. Glashow, in Quark and Leptons, eds. M. Levy et al. (Plenum, NY, 1980); R. N. Mohapatra and G. Senjanovic, Phys. Rev. Lett. 44, 912 (1980).

3. A. Zee, Phys. Lett. B93, 389 (1980); L. Wolfenstein, Nucl. Phys. B175, 92 (1980); S. T. Petcov, Phys. Lett. B115, 401 (1982).

4. C. G. Callen, S. Coleman, J. Wess, and B. Zumino, Phys. Rev. 177, 2247 (1969);

S. Weinberg, Physica A96, 327 (1979).

5. D. A. Dicus and V. S. Mathur, Phys. Rev. D7, 3111 (1973).

6. B. W. Lee, C. Quigg, H. B. Thacker, Phys. Rev. D16, 1519 (1977).

7. M. Veltman, Acta. Phys. Polon. B8, 475 (1977).

8. M. S. Chanowitz and M. K. Gaillard, Nuc. Phys. B261, 379 (1985).

9. T. Appelquist and M. S. Chanowitz, Phys. Rev. Lett. 59, 2405 (1987).

10. F. Maltoni, J. M. Niczyporuk, S. Willenbrock, Phys. Rev. Lett. 86, 212 (2001).

11. F. Maltoni, J. M. Niczyporuk, S. Willenbrock, Phys. Rev. D65, 033004 (2002).

12. D. A. Dicus and H.-J. He, hep-ph/0409131

13. Super-Kamiokande Collaboration, S. Fukuda, et al., Phys. Rev. Lett. 86, 5656 (2001); Y. Ashie, et al., Phys. Rev. Lett. 93, 101801 (2004) hep-ex/0404034.

14. H.-J. He and W. B. Kilgore, Phys. Rev. D55, 1515 (1997); H.-J. He, Y.-P. Kuang, C.-P. Yuan, Phys. Rev. D51, 6463 (1995); H.-J. He, Y.-P. Kuang, X. Li, Phys. Lett. B329, 278 (1994); Phys. Rev. D49, 4842 (1994); Phys. Rev. Lett. 69, 2619 (1992); and references therein.

15. H.-J. He, Y.-P. Kuang and C.-P. Yuan, Phys. Rev. D55, 3038 (1997); Phys. Lett. B382, 149 (1996); and a comprehensive review, DESY-97-056 hep-ph/9704276.

16. T. Appelquist and J. Carrazone, Phys. Rev. D11, 2856 (1975). 